\title{
A MATHEURISTIC APPROACH FOR THE MAXIMUM BALANCED SUBGRAPH OF A SIGNED GRAPH
}

\author{
Jorge Reynaldo Moreno Ramírez*๑๐, Yuri Abitbol de Menezes Frota \\ and Simone de Lima Martins
}

\begin{abstract}
A graph $G=(V, E)$ with its edges labeled in the set $\{+,-\}$ is called a signed graph. It is balanced if its set of vertices $V$ can be partitioned into two sets $V_{1}$ and $V_{2}$, such that all positive edges connect nodes within $V_{1}$ or $V_{2}$, and all negative edges connect nodes between $V_{1}$ and $V_{2}$. The maximum balanced subgraph problem (MBSP) for a signed graph is the problem of finding a balanced subgraph with the maximum number of vertices. In this work, we present the first polynomial integer linear programming formulation for this problem and a matheuristic to obtain good quality solutions in a short time. The results obtained for different sets of instances show the effectiveness of the matheuristic, optimally solving several instances and finding better results than the exact method in a much shorter computational time.
\end{abstract}

Mathematics Subject Classification. 90C59, 90C27, 90C05, 90C10.

Received January 25, 2021. Accepted September 20, 2021.

\section{INTRODUCTION}

Let $G=(V, E)$ be an undirected graph where $V$ is the set of vertices and $E$ is the set of edges. Consider the set of labels $\{+,-\}$ and the function $s: E \rightarrow\{+,-\}$ that assigns a label (or sign) to each edge in $E$. An edge $e \in E$ is called negative if $s(e)=-$ and positive if $s(e)=+$. For some interesting problems an edge can be both positive and negative. In this case, it is called a parallel edge $(s(e)= \pm)$. In this context, an undirected graph $G$ together with a function $s$ is called a signed graph $G=(V, E, s)$ or briefly an s-graph.

Signed graphs were introduced by Heider [13] for describing sentiment relations between people of the same social group. In the conventional approach for modeling a social network, a positive edge represents a friendship or similarity, while a negative edge represents some enmity. Signed graphs have been studied by researchers in different scientific areas, including portfolio analysis in risk management [12,14], biological systems [5] and detection of embedded matrix structures [11].

The concept of balanced signed graphs was formalized by Cartwright and Harary [4], as an extension of Heider's theory. The authors stated that a balanced signed graph could be partitioned into two vertex sets, being that all edges within the sets are positive, and all those between the sets are negative. It is clear that not every signed graph is balanced, and there are different situations in which finding a balanced signed subgraph

Keywords. Balanced signed graph, local search, matheuristic.

Computer Science Department, Universidade Federal Fluminense, Niterói, RJ, Brazil

*Corresponding author: jorge85.mail@gmail.com 
results in a problem of interest. Despite this fact, the problem of finding a balanced subgraph with maximum number of vertices (MBSP) has been applied in almost as many applications as the problem of finding signed graphs $[11,12,14]$.

To formally define the MBSP problem, we present some additional notations used in the rest of the paper. Let $G=(V, E, s)$ denote a signed graph where $E^{-} \subseteq E$ and $E^{+} \subseteq E$ denote, respectively, the set of negative and positive edges in $G$. Similarly, for a vertex $v \in V$, we define by $\delta^{-}(v)$ and $\delta^{+}(v)$ the set of vertices adjacent to $v$ connected by negative and positive edges. Moreover, for a vertex set $V^{\prime} \subseteq V$, let $E\left[V^{\prime}\right]=\left\{\{u, v\} \in E \mid u, v \in V^{\prime}\right\}$ be the subset of edges induced by $V^{\prime}$ and $G_{V^{\prime}}=\left(V^{\prime}, E\left[V^{\prime}\right], s\right)$ its vertex induced subgraph of $G$. To simplify notation we will assume that $\left|G_{V^{\prime}}\right|=\left|V^{\prime}\right|$. We also define that a signed graph $G=(V, E, s)$ is balanced if its vertex set can be partitioned into sets $V_{1}$ and $V_{2}$ in such a way that $E\left[V_{1}\right] \cup E\left[V_{2}\right]=E^{+}$. Thus, given a signed graph $G=(V, E, s)$, the MBSP is the problem of finding a subset of vertices $V^{\prime} \subseteq V$ such that the subgraph $G_{V^{\prime}}$ is balanced and maximizes the cardinality of $V^{\prime}$.

Gülpinar et al. [11] proved that MBSP is NP-hard, and the literature presents some heuristic and exact approaches to solve this problem $[8,9,11]$. Branch-and-cut methods presented in $[8,9]$ use mathematical models based on the fact that a signed graph is balanced if and only if it does not contain a parallel edge or a cycle with an odd number of negative edges $[2,11,21]$. Also, heuristics and metaheuristics were developed for this problem, such as the GGMZ heuristic [11] and a GRASP metaheuristic in [8]. More recently, a new heuristic for this problem, based on negative chordless cycles, was presented by Marinelli and Parente [16].

Different strategies appear in the literature to combine exact and heuristics algorithms. In this context, matheuristics $[7,10,17]$ have attracted the attention of the scientific community. Matheuristics may use some features of the mathematical models of one problem to customize heuristics to solve these problems or use heuristics to improve time effectiveness of mathematical programming techniques.

In this work, we propose an alternative optimization formulation for the one proposed in $[8,9]$. In contrast with their formulation, the size of our optimization model is polynomial in the input size. We also introduce a matheuristic developed to solve the MBSP to obtain good quality solutions quickly.

The remainder of this paper is organized as follows. Section 2 presents a new formulation for the MBSP problem. Section 3 describes the main algorithms used in the proposed matheuristic. In Section 4, we present the results obtained by the matheuristic and the comparison with previous heuristics. Finally, conclusions are discussed in Section 5.

\section{A NEW FORMulation FOR THE MBSP}

Previous mathematical formulations are based in the fact that a signed graph is balanced if and only if it does not contain a parallel edge or a cycle with an odd number of negative edges. The branch-and-cut algorithm proposed in [9] and extended in [8] is based on this result and consider a possible exponential set of odd negative cycles in $G$, denoted as $C^{-}$.

The formulation used in this algorithm is the following:

$$
\max \sum_{v \in V} y_{v}
$$

subject to:

$$
\begin{aligned}
y_{u}+y_{v} \leq 1, & \forall\{u, v\} \in E^{-} \cap E^{+}, \\
\sum_{v \in C} y_{v} \leq|C|-1, & \forall C \in C^{-} \\
y_{v} \in\{0,1\}, & \forall v \in V
\end{aligned}
$$

where $y_{v}=1$ if and only if vertex $v$ belongs to the balanced subgraph, $y_{v}=0$ otherwise. The objective function (2.1) tries to maximize the number of vertices in the balanced subgraph. Constraints (2.2) ensure that the 
endpoints vertices $u$ and $v$ of any parallel edge cannot belong together to the balanced subgraph. Constraints (2.3), denoted as odd negative cycle inequalities, prohibit cycles with an odd number of negative edges in the subgraph.

In this work, a different formulation is proposed based on the construction of two disjoint sets $V_{1}, V_{2} \subseteq V$, aiming to maximize the number of vertices in the resulting balanced signed graph $G_{V_{1} \cup V_{2}}$. Our main objective is to employ a polynomial number of constraints in order to reach a polynomial bounded formulation. Thus, we define the binary variables $x_{v}$ for all $v \in V$, such that $x_{v}=1$ if and only if vertex $v$ belongs to the set $V_{1}$; otherwise, $x_{v}=0$. Similarly, for all $v \in V$, we define binary variables $z_{v}=1$ if vertex $v$ belongs to the set $V_{2}$; otherwise, $z_{v}=0$. In this context, we have formulated the MBSP as the following integer programming problem:

$$
\max \sum_{v \in V}\left(x_{v}+z_{v}\right)
$$

subject to:

$$
\begin{aligned}
x_{v}+z_{v} \leq 1, & \forall v \in V, \\
x_{u}+x_{v} \leq 1, & \forall\{u, v\} \in E^{-}, \\
z_{u}+z_{v} \leq 1, & \forall\{u, v\} \in E^{-}, \\
x_{u}+z_{v} \leq 1, & \forall(u, v) \in A^{+}, \\
x_{u}+z_{u}+x_{v}+z_{v} \leq 1, & \forall\{u, v\} \in E^{+} \cap E^{-}, \\
x_{v} \in\{0,1\}, & \forall v \in V, \\
z_{v} \in\{0,1\}, & \forall v \in V,
\end{aligned}
$$

where $A^{+}=\left\{(u, v),(v, u) \mid\{u, v\} \in E^{+}\right\}$.

The above model is said to be a clustering formulation. The objective function (2.5) maximizes the total number of vertices in $V_{1} \cup V_{2}$. Constraints (2.6) indicate that any vertex in the solution has to belong to only one set, $V_{1}$ or $V_{2}$. Constraints (2.7) and (2.8) ensure that there are no negative edges with both endpoints in $V_{1}$ and $V_{2}$, respectively. Moreover, constraints (2.9) indicate that there are no positive edges that have one endpoint in $V_{1}$ and the other in $V_{2}$. Constraints (2.10) ensure that if there is a parallel edge between two vertices, both vertices cannot be in the solution.

\section{A MATheuristic FOR THE MBSP}

In Figueiredo and Frota [8], a GRASP heuristic was proposed to solve the MBSP and provided the best quality results among the heuristics developed for this problem for the instances analyzed. In addition, the $\mathrm{NCCH}$ heuristic presented in [16], obtained the best quality results for the instances used in that work. This section details our proposed matheuristic to tackle the MBSP.

\subsection{The NCCH heuristic}

The greedy heuristic NCCH proposed in [16] is based on the observation that a vertex set $U \subseteq V$ induces a balanced subgraph $G_{U}$ if and only if $G_{U}$ contains no negative cycles, i.e., cycles with an odd number of negative edges [2]. Therefore, this greedy approach tries to reduce negative cycles progressively by applying the Negative Cycle Contraction (NCC) rule shown in Algorithm 1 [16]. This procedure preserves the sign of all negative cycles and shortens those containing an edge $\{u, v\}$ that is not a parallel edge. Any balanced induced subgraph of the signed graph obtained by applying the NCC-rule to all the vertices of $V$ is a balanced induced subgraph of $G$.

Algorithm 2 presents the NCCH heuristic using this rule. 

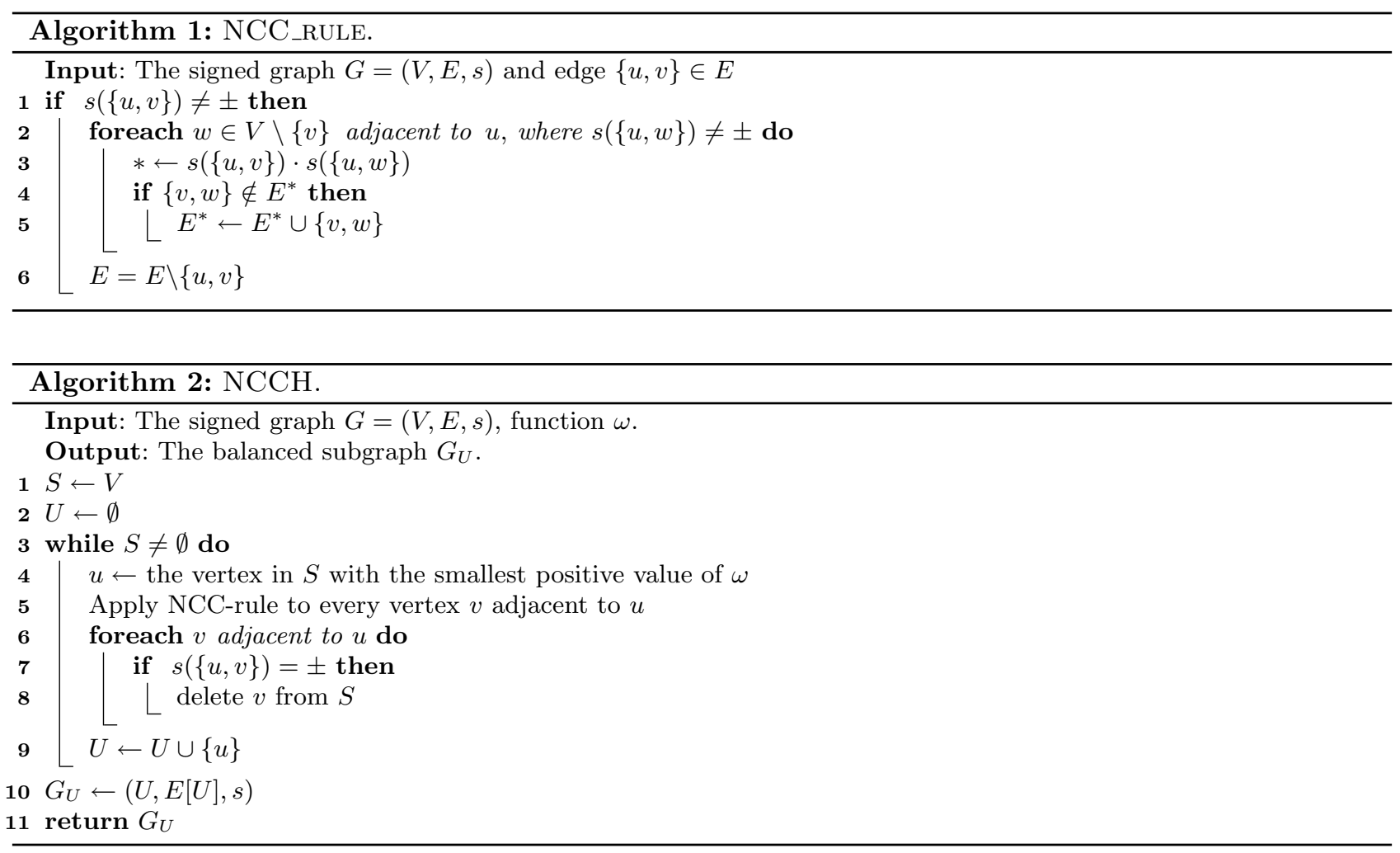

This greedy procedure runs from lines 3 to 9 , while there are vertices from $S$ to be examined. In each step, it selects a vertex $u$ by using a greedy criterion defined by the function $\omega$ (line 4 ). Then it applies the NCC rule to every vertex $v$ adjacent to $u$ (line 5). Later (lines 6-8), the method deletes all parallel edges generated after applying the NCC-rule. At the end of Algorithm 2 (line 10), the vertex set $U$ induces a balanced signed graph (see details in [16]).

The function $\omega$ should prioritize vertices that have few and small negative cycles without chords associated with them. Marinelli and Parente [16] proposed three options for $\omega$, and showed that the performance of the heuristic varies depending on the $\omega$ function and instance structure.

\subsection{Construction procedure based on negative chordless cycles}

Based on the NCCH heuristic, we developed a construction procedure using a Restricted Candidate List (RCL) [19]. This procedure, shown in Algorithm 3, is similar to the above algorithm. The only difference between the original $\mathrm{NCCH}$ heuristic and this new one is that the former greedily selects the vertex using the function $\omega$, while the latter creates a list $L$ to select the vertex from that list randomly. The list $L$ contains all vertices with the minimum value for the $\omega$ function.

Choosing a random vertex from this list at each step causes the final solution to differ from the solution obtained by the NCCH heuristic. Thus, Algorithm 3 can be used to generate different feasible solutions. In this work $\omega(v)=d_{G}(v)$ was selected, where $d_{G}(v)$ represents the degree of vertex $v \in V$. This is one of the functions proposed in [16], and it was chosen because it demands less computational time to be calculated. 


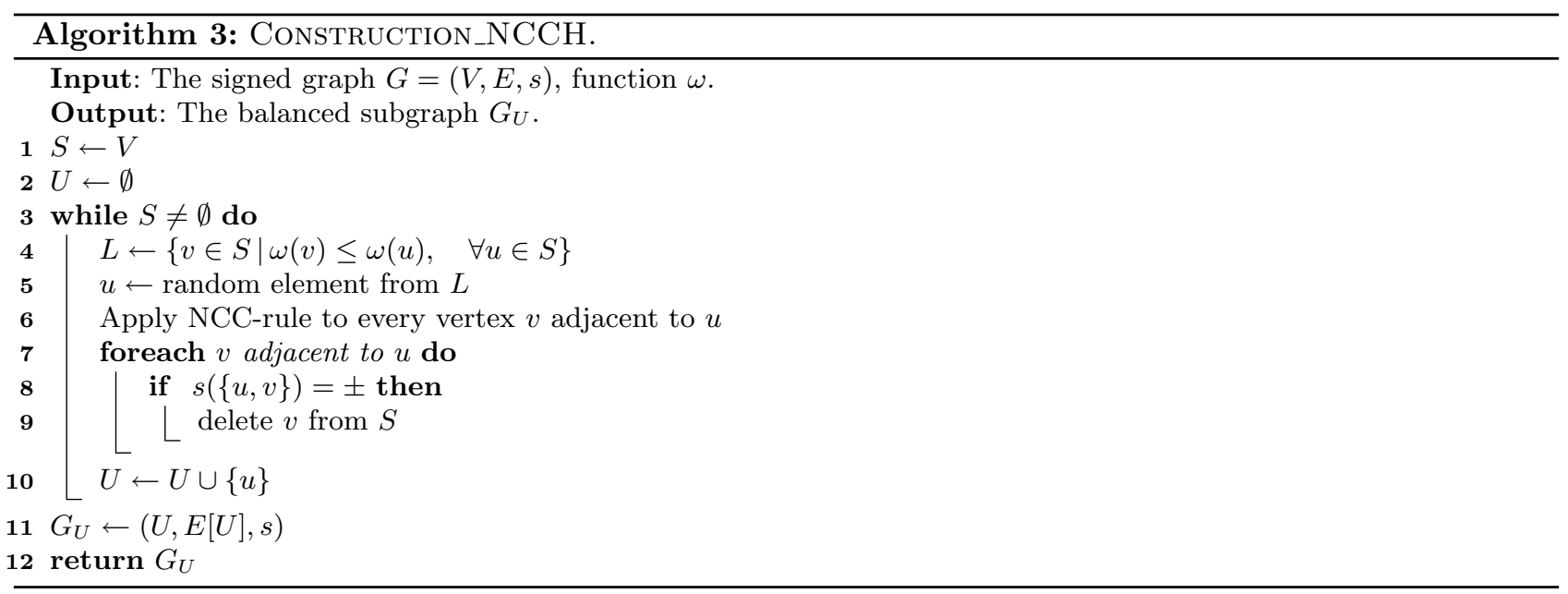

\subsection{Local Search}

The purpose of this procedure is to find locally optimal solutions with respect to some neighborhood in order to improve the initial solution provided by Algorithm 3. The local search in [8] obtains neighboring solutions by removing one or two vertices from the current solution, and including as many vertices as possible (maintaining feasibility), in order to reach an improved solution.

If we consider the removal of all possible pairs of elements, a quadratic component is incorporated in the local search. This neighborhood would be computationally expensive in large graphs. So, our local search procedure obtains neighboring solutions by randomly removing $p \leq\left|U^{\prime}\right|$ vertices from a feasible solution $G_{U^{\prime}}=$ $\left(U^{\prime}, E\left[U^{\prime}\right], s\right)$ (shrinking phase) and reapplying the $\mathrm{NCCH}$ heuristic shown in Algorithm 2 (expansion phase). The parameter $p$ is determined by the input parameter $\alpha$ that indicates a percentage of the number of vertices of the input graph $G$ (line 2). Thereby, we define the $p$-neighborhood, denoted by $N_{p}$, as the family of all solutions obtained by removing $p$ vertices from $U^{\prime}$ and reapplying the $\mathrm{NCCH}$ heuristic described in Algorithm 2. As the number of neighbors grows exponentially, only $k$ neighbors in $N_{p}$ are analyzed in each local search step.

The local search procedure, shown in Algorithm 4, tries to increase the solution's cardinality returned by the construction phase. Each time it finds an enhancement, the current best solution $G_{\text {best }}$ is updated (lines 6-8). The method stops if there is no further improvement of the solution after exploring $k$ neighbors in $N_{p}$.

\subsection{The MS_NCCH heuristic}

Using the constructive procedure and the local search algorithm we propose the MS_NCCH heuristic described in Algorithm 5, based on a multi-start schema.

Each MS_NCCH iteration starts with an initial solution generated for the problem (lines 6-9). For the first iteration, we use the greedy NCCH heuristic described in Algorithm 2. Then, we use the constructive procedure defined in Section 3.2 for the remaining iterations. Once a feasible solution is created, the local search tries to improve the quality of that solution (line 10). The best global solution $G_{\text {best }}$ is updated (line 12) if the solution found by the local search improves it. The method maintain a pool of elite solutions $E L$ (line 13) that is updated with new solutions found in the local search procedure that are better than those in $E L$, respecting the size limit EliteSize of $E L$. That implies that all neighboring solutions obtained in the local search stage are considered. As $E L$ has a limited size, when a solution has a better value than the worst solution of $E L$, the new solution enters the set, while the worst solution leaves $E L$.

From lines 14 to 17 the set of elite solutions is checked. If some new solution enters the set, then the counter for the number of iterations without change in $E L$ is restarted. 

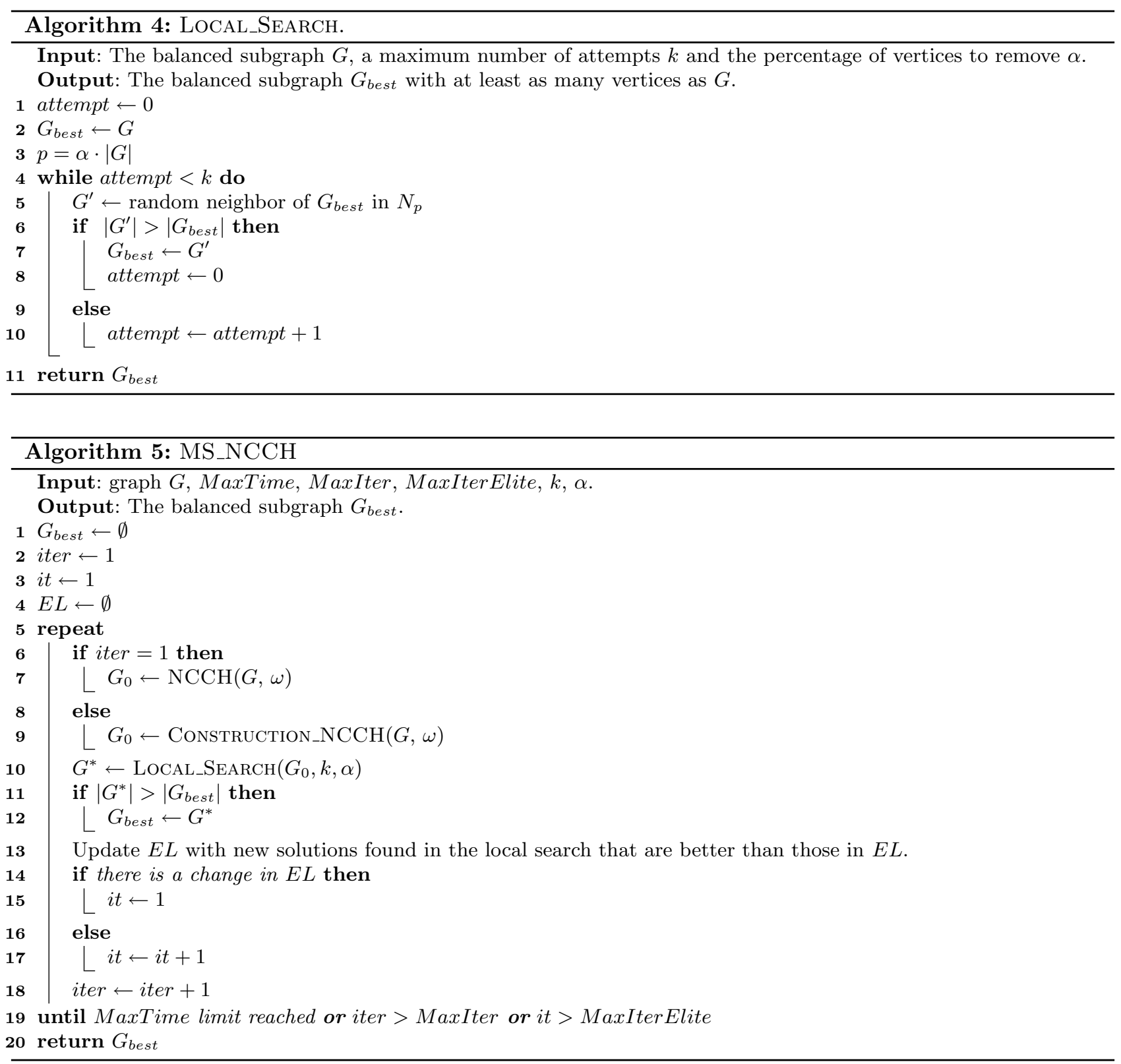

The algorithm stops if it reaches the maximum running time MaxTime, the maximum number of overall iterations MaxIter, or there are no changes in the elite set during MaxIterElite iterations.

\subsection{The matheuristic MH_MBSP}

The MH_MBSP is a matheuristic developed using the proposed formulation presented in Section 2 and the solutions inserted in the elite set by the MS_NCCH heuristic. The proposed compact formulation can be described using a polynomial number of variables and constraints. This feature allows the formulation to be integrated with the MS_NCCH heuristic in order to quickly find feasible solutions after fixing a subset of variables. The quality of solution depends on the choice of which and how many variables will be selected to be fixed. 
In our first approach we try to identify a subset of vertices $U \subseteq V$ that appear in all solutions of the elite set $E L$ in the end of the method MS_NCCH. Then, we include Constraints 3.1 into the formulation, indicating that these vertices must be present in the solution:

$$
x_{v}+z_{v}=1, \quad \forall v \in U .
$$

Unfortunately, this approach turned out to be very restrictive to the formulation, not improving the solutions. So, we decided to use the subset of vertices that appear in all solutions that were ever inserted in the elite set. This strategy improved the results and is adaptive for each instance, as the number of solutions inserted in the elite set varies depending on the instance.

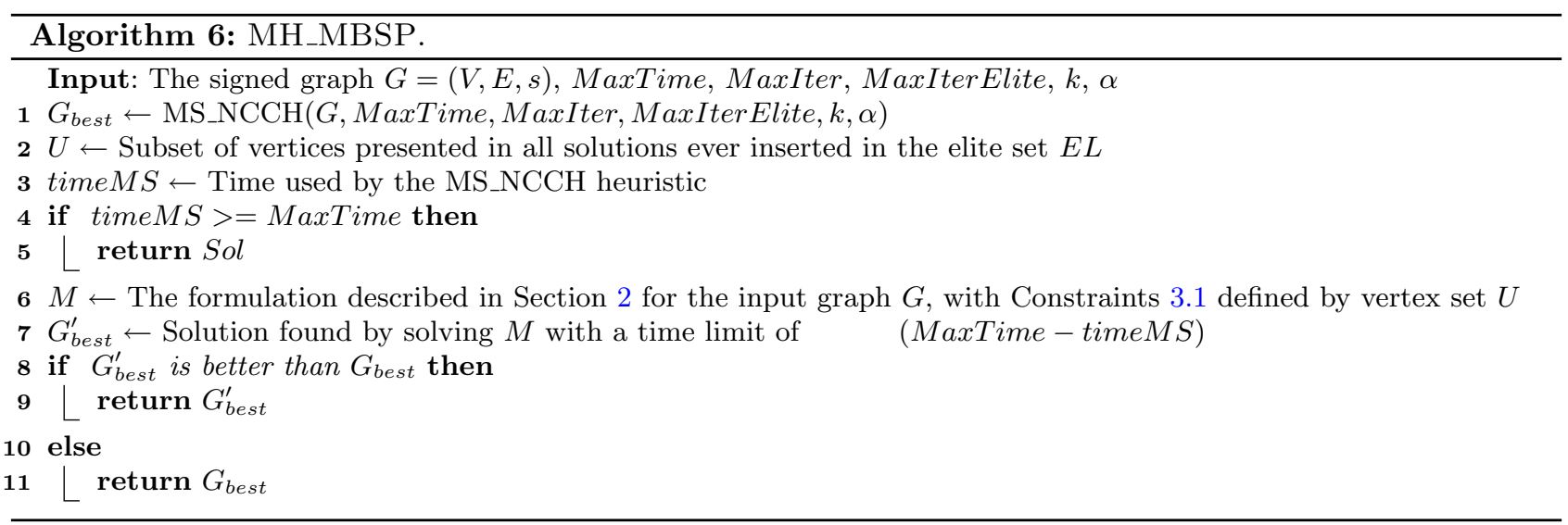

Algorithm 6 shows the MH_MBSP matheuristic for the MBSP problem. It starts by storing the best solution obtained by the MS_NCCH heuristic (line 1) and the subset of vertices presented in all solutions ever inserted in the elite set (line 2).

If the time used by the heuristic reached the time limit MaxTime, it returns the solution found by the MS_NCCH heuristic (line 5); otherwise, the model $M$ is created according to Section 2 (line 6), and expanded by Constraints 3.1 defined by vertex set $U$, indicating that these vertices must be fixed in the solution, belonging to any of solution sets $V_{1}$ or $V_{2}$.

Subsequently, the formulation is solved using (MaxTimeMH-timeMS) as the time limit (line 7). The matheuristic result is the best solution between the one found by the MS_NCCH heuristic and the one found by solving the model with the initialized variables (lines $8-11$ ).

\section{Computational experiments}

This section reports the computational experiments carried out with the heuristics methods described in the previous sections. We performed several computational experiments to validate the effectiveness of the proposed methods. First, we analyzed the same set of instances of [8], which contains several groups of instances (DMERN, Portfolio, and Random) having signed graphs with different sizes and densities. Then, we analyzed the set of instances proposed in [16], which contains three groups of instances (N, P, and R instances).

\subsection{Exact method}

The new proposed mathematical formulation described in Section 2 is the starting point for our exact method used in the matheuristic. We executed it for an hour to verify its performance. Experiments for the exact method were executed on an Intel (R) Core i5-4460S CPU @ $2.90 \mathrm{GHz}$ and $8 \mathrm{~Gb}$ of RAM using the operating system 
Fedora 22. All methods were programmed in $\mathrm{C}++$ language using the gcc compiler. The IBM ILOG CPLEX 12.6 was used with a single thread of execution, and all other CPLEX parameters were left to their default values.

In [8], the authors showed the results obtained by their exact method based on removing cycles with an odd number of negative edges. These experiments were executed on an Intel (R) Pentium (R) 4 CPU $3.06 \mathrm{GHz}$, equipped with $3 \mathrm{~GB}$ of RAM. In order to establish a more similar environment, we calculated the associated time scaling factor of 0.34 (based on Pass mark benchmark), representing the ratio between the speed of the processor in [8] and the speed of our processor. Based on this, we show their time values multiplied by this associated factor.

Table 1 shows the results obtained for instances set DMERN, composed of 61 instances with the number of vertices $|V|$ varying from 144 to 8317 . Columns 2, 3, and 4 present, respectively, the upper bound provided by the root node relaxation, the best lower bound value, and the time to reach this value for the exact model shown in [8]. Columns 5, 6, and 7 have the same meaning, but for our exact method. The symbol "-" means that the optimal solution was not found within the execution time limit.

According to Table 1, our method could solve nine more instances than the method in [8]. On the other hand, the new formulation presents a much weaker relaxation at the root node. However, the resolution times were short, and our exact method presents high-quality lower bounds (feasible solutions). Together with the fact that the new formulation is compact, these features indicate that our proposed formulation may be a good choice to be integrated with a heuristic to search for high-quality and fast feasible solutions.

\subsection{Choosing parameters}

We used the Irace package [15] to select the parameters for the MS_NCCH heuristic. This package includes the iterated F-race algorithm [3] and several extensions for finding the most appropriate parameter settings for an optimization algorithm. It needs the specification of the parameters to be configured and instances for training. According to Section 3.4, the parameters to be configured are:

$-\alpha$ : The percentage of vertices to be removed from the solution set in the local search phase. Its values ranged from $5 \%$ to $50 \%$.

- $k$ : The maximum number of neighbors explored during the local search. Its values were in the range of 10 to 1000 .

- it: The number of iterations without a change in the elite set, tested in the range of 1 to 50 .

We configure the Irace to run 1000 experiments using 10 instances, none of them present in previous works or used in later comparisons. The instances used were:boeing1, boeing2, capri, degen2, file150.7, finnis, scfxm1, scfxm3, scsd6 and standata, coming from a set of general mixed integer programs in Netlib (https://www. netlib.org/lp/data). The instances were selected with different sizes and densities to provide parameters that work well with different input structures.

From the results of Irace, the final configurations were: $\alpha=33.3 \%, k=1000$ and $i t=20$. The parameter EliteSize was set to 10, as used in Martins et al. [18] and de Holanda Maia et al. [6]. For all experiments, we used MaxIter $=100$ and MaxTime $=30 \mathrm{~s}$.

\subsection{Analyzing the performance of the heuristics}

Tests were developed on an Intel(R) Core(TM) i5-7200U CPU @ $2.50 \mathrm{GHz}$ with 8 Gb of RAM using Ubuntu 16.04. All methods were programmed in $\mathrm{C}++$ language using the gcc compiler. We used the instances presented in [16] to analyze the NCCH, MS-NCCH, and MH-MBSP heuristics performance. The goal of these tests was to verify the impact on the solution of each developed strategy. For all heuristics, the function $w$ is the vertex degree.

The three sets of instances are N-instances, P-instances, and R-instances. N-instances consist of 34 signed graphs obtained from a set of preprocessed and scaled Netlib matrices. P-instances are 50 randomly generated 
TABLE 1. Exact results for instance set DMERN.

\begin{tabular}{|c|c|c|c|c|c|c|}
\hline Instance & $\operatorname{Relax}_{C}$ & $\mathrm{ILP}_{C}$ & Time $_{C}$ & Relax & ILP & Time \\
\hline danoint & 126 & 97 & 165.0 & 136 & 97 & 0.5 \\
\hline bienst1 & 118 & 90 & 2772.0 & 184 & 90 & 1.0 \\
\hline stein 45 & 30.17 & 29 & - & 331 & 30 & 0.1 \\
\hline disctom & 397 & 299 & 643.0 & 399 & 299 & 44.0 \\
\hline fc. 60.20 .1 & 384 & 371 & 171.0 & 414 & 371 & 7.3 \\
\hline air05 & 240.5 & 63 & - & 426 & 69 & - \\
\hline neos17 & 2 & 2 & 61.0 & 485 & 2 & 0.4 \\
\hline p100x588 & 638 & 633 & 63.0 & 688 & 633 & 0.4 \\
\hline air04 & 383 & 113 & - & 823 & 133 & - \\
\hline r80x800 & 840 & 828 & 699.0 & 880 & 828 & 19.1 \\
\hline nug08 & 301 & 128 & 150.0 & 912 & 128 & 0.2 \\
\hline p50x864 & 889 & 884 & 107.0 & 914 & 884 & 4.4 \\
\hline n5-3 & 941 & 912 & 82.0 & 1012 & 912 & 0.3 \\
\hline neos 21 & 924 & 191 & 782.0 & 1085 & 191 & 0.4 \\
\hline $\mathrm{n} 4-3$ & 1109 & 1062 & 165.0 & 1178 & 1062 & 0.7 \\
\hline dano3mip & 1150 & 588 & - & 1203 & 760 & - \\
\hline n8-3 & 1197 & 1176 & 120.0 & 1300 & 1176 & 0.3 \\
\hline neos 20 & 595.5 & 627 & 107.0 & 1320 & 627 & 0.1 \\
\hline p200x1188 & 1288 & 1272 & - & 1388 & 1273 & - \\
\hline $\mathrm{p} 200 \times 1188 \mathrm{c}$ & 1288 & 1273 & - & 1388 & 1276 & 365.1 \\
\hline roll3000 & 583 & 824 & 170.0 & 1205 & 824 & 0.9 \\
\hline janos-us-ca-D-D-M-N-C-A-N-N & 1554 & 1521 & 214.0 & 1643 & 1521 & 0.5 \\
\hline pioro40-D-B-M-N-C-A-N-N & 1573 & 1560 & 126.0 & 1649 & 1560 & 0.5 \\
\hline n13-3 & 1562 & 1537 & 214.0 & 1661 & 1537 & 0.7 \\
\hline $\mathrm{n} 2-3$ & 1669 & 1656 & 259.0 & 1752 & 1656 & 0.7 \\
\hline zib54-U-U-E-N-C-A-N-N & 1728 & 1728 & 62.0 & 1728 & 1728 & 0.5 \\
\hline qap10 & 200 & 200 & 427.0 & 1820 & 200 & 1.9 \\
\hline ns1688347 & 468.43 & 375 & - & 1806 & 448 & - \\
\hline germany50-U-U-M-N-C-A-N-N & 2000 & 2000 & 91.0 & 2000 & 2000 & 0.4 \\
\hline protfold & 611.61 & 383 & - & 1990 & 574 & - \\
\hline cap6000 & 2074 & 2074 & 111.0 & 2169 & 2074 & 103.7 \\
\hline $\mathrm{n} 7-3$ & 2197 & 2162 & 1191.0 & 2278 & 2162 & 2.4 \\
\hline n9-3 & 2187 & 2112 & 1330.0 & 2280 & 2112 & 4.6 \\
\hline acc-1 & 567 & 480 & - & 2286 & 491 & 41.3 \\
\hline n3-3 & 2192 & 2059 & 2827.0 & 2303 & 2059 & 8.5 \\
\hline zib54-D-B-E-N-C-A-N-N & 2277 & 2268 & 211.0 & 2347 & 2268 & 0.8 \\
\hline n12-3 & 2265 & 2214 & 1067.0 & 2358 & 2214 & 3.3 \\
\hline neos 818918 & 2019.83 & 2018 & 800.0 & 2350 & 2018 & 6.2 \\
\hline germany50-D-B-M-N-C-A-N-N & 2355 & 2350 & 262.0 & 2438 & 2350 & 0.8 \\
\hline acc-2 & 567 & 454 & - & 2520 & 491 & 60.0 \\
\hline ta2-U-U-M-N-C-A-N-N & 2470 & 2471 & 175.0 & 2470 & 2471 & 0.5 \\
\hline n6-3 & 2602 & 2538 & 2785.0 & 2686 & 2538 & 5.1 \\
\hline berlin & 2678 & 2653 & - & 2704 & 2653 & 44.4 \\
\hline neos11 & 752.11 & 702 & - & 2686 & 742 & 3.3 \\
\hline ta2-D-B-M-N-C-A-N-N & 2748 & 2731 & 470.0 & 2837 & 2731 & 2.9 \\
\hline acc- 6 & 1011.28 & 899 & - & 3040 & 939 & - \\
\hline acc-5 & 1002.93 & 875 & - & 3045 & 944 & - \\
\hline acc-3 & 1125 & 1125 & 221.0 & 3240 & 1071 & - \\
\hline
\end{tabular}


TABLE 1. continued.

\begin{tabular}{lrrrrrr}
\hline \hline Instance & Relax $_{C}$ & ILP $_{C}$ & Time $_{C}$ & Relax & ILP & Time \\
\hline acc-4 & 1125 & 1125 & 240.0 & 3276 & 1071 & - \\
brasil & 3335 & 3307 & - & 3364 & 3307 & 32.6 \\
mkc & 2964 & 3220 & 341.0 & 3124 & 3223 & 0.5 \\
p500x2988 & 3238 & 3199 & - & 3488 & 3206 & - \\
p500x2988c & 3238 & 3098 & - & 3488 & 3194 & - \\
mod011 & 3208 & 3854 & 416.0 & 3240 & 3854 & 0.8 \\
neos1 & 1194 & 1099 & - & 2712 & 1186 & 29.3 \\
seymour & 472.04 & 559 & - & 4794 & 610 & - \\
seymour1 & 472.04 & 599 & - & 4794 & 610 & - \\
n370a & 5100 & 5100 & 1374.0 & 5150 & 5100 & 1.4 \\
rentacar & 3280 & 4662 & 2403.0 & 4257 & 4693 & 1.5 \\
manna81 & 3382 & 2322 & 1178.0 & 6480 & 2322 & - \\
neos12 & 1546.41 & 1401 & - & 8275 & 1546 & - \\
\hline
\end{tabular}

power-law signed graphs, five instances for each size $|V|=100 k$ with $k \in\{1, \ldots, 10\}$. R-instances are random signed graphs generated with the function Random $\operatorname{Graph}(n, p)$ provided by Mathematica [20], with the number of vertices ranging from 50 to 200 and density in the set $\{0.1,0.3,0.5,0.7\}$.

Table 2 shows the results for N-instances. Column 2 shows the results of the greedy heuristic NCCH. The time in seconds, used by this heuristic, is presented in Column 3. Columns 4 and 5 show the average result and time of MS-NCCH heuristic, respectively, after 10 runs. Next two columns show the same information but for the MH-MBSP matheuristic. The final column shows the gap between the best solution found by the exact method (ILP) and the average result found by the matheuristic (Col. 6). The gap is calculated by the following equation:

$$
\text { gap }=\text { Best }_{\mathrm{ILP}}-\operatorname{Avg}_{\mathrm{MH}-\mathrm{MBSP}}
$$

where Best ${ }_{\text {ILP }}$ is the best solution found by our exact method in the time limit of $1 \mathrm{~h}$.

Since the exact optimization phase of the matheuristic starts when the MS-NCCH heuristic finishes, the results and time of MS-NCCH are always worse and less than or equal to those obtained by MH-MBSP. For the same reason, the NCCH results are always worse than or equal to those obtained by the MS-NCCH heuristic. Cells with an asterisk in the last column indicate that the problem was not optimally solved in $1 \mathrm{~h}$ and the gap is calculated related to the best feasible solution found. Moreover, the value "-" in the time column indicates that the method reached the maximum assigned time of $30 \mathrm{~s}$.

The N-instances set contains "easy" instances, and the greedy heuristic NCCH was able to solve optimally 22 of the 34 instances. For those 12 instances not solved optimally by the NCCH heuristic, the MS-NCCH heuristic solved optimally eight new instances and improved the results for other two unsolved instances. The exact optimization phase of the matheuristic did not improve the results of the remaining four unsolved instances. When analyzing these instances, we can see that they are instances in which the MS-NCCH heuristic used all execution time, and there was no available time to perform the exact optimization phase in three of them.

Table 3 shows the results for P-instances. Columns of this table have the same meaning as in Table 2. These instances are a little more complex than N-instances, and the results reflect that. In this set, the NCCH heuristic found seven optimal results. For the remaining 43 instances, the MS-NCCH heuristic always obtained a better result than the NCCH heuristic and found 10 additional optimal solutions. For the remaining 33 unsolved instances, the matheuristic MH-MBSP improved all results and found 19 new optimal values.

The R-instances are classified in [16] as hard instances because they are graphs connected with many negative cycles. Table 4 shows the results for these instances, and its columns have the same meaning as in the previous tables. The MS-NCCH heuristic improved all results found by the NCCH heuristic. Moreover, the MS-NCCH 
TABLE 2. Heuristics results for N-instances.

\begin{tabular}{|c|c|c|c|c|c|c|c|}
\hline \multirow[b]{2}{*}{ Instance } & \multicolumn{2}{|c|}{$\mathrm{NCCH}$} & \multicolumn{2}{|c|}{ MS-NCCH } & \multicolumn{3}{|c|}{ MH-MBSP } \\
\hline & Result & Time & Avg & Time & Avg & Time & Gap \\
\hline $25 \mathrm{fv} 47$ & 212 & 0.00 & 212.0 & 0.22 & 212.0 & 0.23 & 0.00 \\
\hline 80bau3b & 1328 & 0.01 & 1329.1 & 12.72 & 1329.1 & 12.76 & 16.90 \\
\hline agg2 & 62 & 0.00 & 62.0 & 0.09 & 62.0 & 0.10 & 0.00 \\
\hline agg3 & 62 & 0.00 & 62.0 & 0.09 & 62.0 & 0.10 & 0.00 \\
\hline bnl1 & 261 & 0.00 & 261.0 & 0.23 & 261.0 & 0.24 & 0.00 \\
\hline bnl2 & 1364 & 0.01 & 1367.0 & 1.25 & 1367.0 & 1.28 & 0.00 \\
\hline cycle & 504 & 0.00 & 504.0 & 1.06 & 504.0 & 1.06 & 0.00 \\
\hline czprob & 717 & 0.00 & 717.0 & - & 717.0 & - & 1.00 \\
\hline $\mathrm{d} 2 \mathrm{q} 06 \mathrm{c}$ & 802 & 0.00 & 804.0 & 0.45 & 804.0 & 0.47 & 0.00 \\
\hline degen 3 & 776 & 0.01 & 796.0 & 2.83 & 796.0 & 3.39 & 0.00 \\
\hline dfl001 & 4175 & 0.57 & 4409.1 & - & 4409.1 & - & *103.90 \\
\hline fffff 800 & 125 & 0.00 & 125.0 & 0.29 & 125.0 & 0.30 & 0.00 \\
\hline ganges & 534 & 0.00 & 534.0 & 1.96 & 534.0 & 1.98 & 0.00 \\
\hline gfrd-pnc & 522 & 0.00 & 522.0 & 1.77 & 522.0 & 1.82 & 0.00 \\
\hline greenbea & 884 & 0.00 & 890.0 & 3.92 & 890.0 & 3.95 & 0.00 \\
\hline greenbeb & 884 & 0.00 & 890.0 & 3.96 & 890.0 & 3.98 & 0.00 \\
\hline maros & 300 & 0.00 & 300.0 & 0.37 & 300.0 & 0.38 & 0.00 \\
\hline modszk1 & 130 & 0.00 & 130.0 & 0.02 & 130.0 & 0.02 & 0.00 \\
\hline nesm & 190 & 0.00 & 190.0 & 0.17 & 190.0 & 0.17 & 0.00 \\
\hline perold & 143 & 0.00 & 143.0 & 0.07 & 143.0 & 0.08 & 0.00 \\
\hline pilot87 & 306 & 0.00 & 306.0 & 0.39 & 306.0 & 0.40 & 0.00 \\
\hline pilot & 252 & 0.00 & 252.0 & 0.24 & 252.0 & 0.24 & 0.00 \\
\hline pilot.ja & 198 & 0.00 & 198.0 & 0.13 & 198.0 & 0.14 & 0.00 \\
\hline pilotnov & 203 & 0.00 & 203.0 & 0.13 & 203.0 & 0.13 & 0.00 \\
\hline pilot.we & 202 & 0.00 & 202.0 & 0.11 & 202.0 & 0.12 & 0.00 \\
\hline scfxm2 & 250 & 0.00 & 252.0 & 0.03 & 252.0 & 0.05 & 0.00 \\
\hline sctap2 & 470 & 0.00 & 470.0 & 0.84 & 470.0 & 0.84 & 0.00 \\
\hline seba & 140 & 0.00 & 140.0 & 1.04 & 140.0 & 1.24 & 0.00 \\
\hline shell & 480 & 0.00 & 482.0 & 4.13 & 482.0 & 4.15 & 0.00 \\
\hline ship12 & 732 & 0.00 & 732.0 & 6.14 & 732.0 & 6.23 & 0.00 \\
\hline ship12s & 360 & 0.00 & 360.0 & 1.31 & 360.0 & 1.34 & 0.00 \\
\hline stocfor2 & 1104 & 0.01 & 1106.0 & 3.07 & 1106.0 & 3.11 & 0.00 \\
\hline stocfor3 & 8512 & 0.42 & 8513.2 & - & 8513.2 & - & 2.80 \\
\hline woodw & 301 & 0.00 & 301.0 & 0.24 & 301.0 & 0.24 & 0.00 \\
\hline
\end{tabular}

heuristic found 27 optimal solutions. The exact optimization phase of the matheuristic improved the MS-NCCH heuristic results in 14 instances and found six new optimal solutions. The MH-MBSP average results improved the NCCH results by 31.0\%. A remarkable result of the matheuristics MH-MBSP was for instance rn200d05-2, as it obtained, in $7.65 \mathrm{~s}$, a better result than the exact method in one hour of processing.

The above experiments showed the impact that each NCC-rule-based heuristic has on the quality of the solutions. The next section compares the MH-MBSP matheuristic with the heuristic GRASP developed in [8], which is the best-known metaheuristic for the problem.

\subsection{DMERN, portfolio and random graphs}

This section compares the MH-MBSP matheuristic to the GRASP method proposed in [8], which is the best metaheuristic in the literature for the problem. As the authors kindly provided the GRASP source code, we 
TABLE 3. Heuristics results for P-instances.

\begin{tabular}{|c|c|c|c|c|c|c|c|}
\hline \multirow[b]{2}{*}{ Instance } & \multicolumn{2}{|c|}{$\mathrm{NCCH}$} & \multicolumn{2}{|c|}{ MS-NCCH } & \multicolumn{3}{|c|}{ MH-MBSP } \\
\hline & Result & Time & Avg & Time & Avg & Time & Gap \\
\hline g0100_1 & 93 & 0.00 & 94.0 & 0.06 & 94.0 & 0.08 & 0.00 \\
\hline g0100_2 & 94 & 0.00 & 95.0 & 0.05 & 95.0 & 0.06 & 0.00 \\
\hline g0100_3 & 96 & 0.00 & 97.0 & 0.02 & 97.0 & 0.03 & 0.00 \\
\hline g0100_4 & 95 & 0.00 & 95.0 & 0.05 & 95.0 & 0.05 & 0.00 \\
\hline g0100_5 & 94 & 0.00 & 94.0 & 0.04 & 94.0 & 0.05 & 0.00 \\
\hline g0200_1 & 188 & 0.00 & 190.0 & 0.02 & 190.0 & 0.04 & 0.00 \\
\hline g0200_2 & 188 & 0.00 & 190.0 & 0.03 & 190.0 & 0.13 & 0.00 \\
\hline g0200_3 & 188 & 0.00 & 188.0 & 0.01 & 188.0 & 0.03 & 0.00 \\
\hline g0200_4 & 190 & 0.00 & 190.0 & 0.12 & 190.0 & 0.13 & 0.00 \\
\hline g0200_5 & 189 & 0.00 & 190.0 & 0.04 & 190.0 & 0.07 & 0.00 \\
\hline g0300_1 & 281 & 0.00 & 283.9 & 0.11 & 284.0 & 0.27 & 0.00 \\
\hline g0300_2 & 281 & 0.00 & 283.5 & 0.05 & 284.0 & 0.41 & 0.00 \\
\hline g0300_3 & 283 & 0.00 & 285.0 & 0.09 & 285.0 & 0.24 & $0.0 \mathrm{c}$ \\
\hline g0300_4 & 283 & 0.00 & 283.0 & 0.31 & 283.0 & 0.33 & 0.00 \\
\hline g0300_5 & 283 & 0.00 & 285.0 & 0.04 & 285.0 & 0.10 & 0.00 \\
\hline g0400_1 & 377 & 0.00 & 379.7 & 0.08 & 380.0 & 0.41 & 0.00 \\
\hline g0400_2 & 379 & 0.00 & 380.0 & 0.26 & 380.0 & 0.51 & 0.00 \\
\hline g0400_3 & 377 & 0.00 & 378.5 & 0.11 & 379.0 & 0.52 & 0.00 \\
\hline g0400_4 & 381 & 0.00 & 381.0 & 0.20 & 381.0 & 0.23 & 0.00 \\
\hline g0400_5 & 378 & 0.00 & 380.0 & 0.10 & 380.0 & 0.27 & 0.00 \\
\hline g0500_1 & 471 & 0.00 & 474.8 & 0.12 & 475.0 & 0.67 & 0.00 \\
\hline g0500_2 & 473 & 0.00 & 476.6 & 0.16 & 477.0 & 0.47 & 0.00 \\
\hline g0500_3 & 470 & 0.00 & 472.4 & 0.15 & 473.0 & 1.23 & 0.00 \\
\hline g0500_4 & 473 & 0.00 & 473.0 & 0.16 & 473.0 & 0.50 & 0.00 \\
\hline g0500_5 & 476 & 0.00 & 476.9 & 0.13 & 477.0 & 0.27 & 0.00 \\
\hline g0600_1 & 564 & 0.00 & 567.5 & 0.27 & 568.0 & 6.36 & 0.00 \\
\hline g0600_2 & 569 & 0.00 & 571.2 & 0.24 & 571.9 & 1.40 & 0.10 \\
\hline g0600_3 & 566 & 0.00 & 566.8 & 0.25 & 568.0 & 12.86 & $0.0 \mathrm{c}$ \\
\hline g0600_4 & 567 & 0.00 & 568.3 & 0.21 & 569.0 & 2.26 & 0.00 \\
\hline g0600_5 & 567 & 0.00 & 570.6 & 0.24 & 571.0 & 0.80 & 0.00 \\
\hline g0700_1 & 661 & 0.00 & 665.5 & 0.39 & 666.0 & 2.00 & 0.00 \\
\hline g0700_2 & 658 & 0.00 & 667.5 & 0.33 & 668.0 & 1.27 & 0.00 \\
\hline g0700_3 & 661 & 0.00 & 662.5 & 0.29 & 663.0 & 3.30 & 0.00 \\
\hline g0700_4 & 657 & 0.00 & 661.0 & 0.33 & 662.0 & 1.77 & 0.00 \\
\hline g0700_5 & 664 & 0.00 & 664.0 & 0.36 & 664.5 & 0.82 & 0.50 \\
\hline g0800_1 & 750 & 0.00 & 754.6 & 0.44 & 756.6 & 16.98 & 0.40 \\
\hline g0800_2 & 751 & 0.00 & 756.5 & 0.44 & 758.9 & - & 0.10 \\
\hline g0800_3 & 759 & 0.00 & 761.9 & 0.46 & 763.0 & 2.52 & 0.00 \\
\hline g0800_4 & 757 & 0.00 & 758.6 & 0.52 & 759.0 & 6.15 & 0.00 \\
\hline g0800_5 & 754 & 0.00 & 759.3 & 0.39 & 760.0 & 4.15 & 0.00 \\
\hline g0900_1 & 844 & 0.00 & 849.6 & 0.58 & 851.7 & - & 0.30 \\
\hline g0900_2 & 845 & 0.00 & 850.5 & 0.57 & 852.8 & - & 0.20 \\
\hline g0900_3 & 848 & 0.00 & 852.8 & 0.48 & 854.4 & 14.52 & 0.60 \\
\hline g0900_4 & 846 & 0.00 & 849.5 & 0.59 & 852.8 & 20.59 & 0.20 \\
\hline g0900_5 & 848 & 0.00 & 851.3 & 0.60 & 852.9 & - & 0.10 \\
\hline g1000_1 & 937 & 0.00 & 942.0 & 0.59 & 945.2 & - & ${ }^{*} 0.80$ \\
\hline g1000_2 & 941 & 0.00 & 946.8 & 0.59 & 949.0 & - & $* 1.00$ \\
\hline g1000_3 & 940 & 0.00 & 944.6 & 0.68 & 947.3 & - & ${ }^{*} 0.7 \mathrm{C}$ \\
\hline g1000_4 & 942 & 0.00 & 947.2 & 0.80 & 948.7 & 26.93 & 0.30 \\
\hline g1000_5 & 945 & 0.00 & 948.5 & 0.51 & 949.8 & 2.58 & 0.20 \\
\hline
\end{tabular}


TABLE 4. Heuristics results for R-instances.

\begin{tabular}{|c|c|c|c|c|c|c|c|}
\hline \multirow[b]{2}{*}{ Instances } & \multicolumn{2}{|c|}{$\mathrm{NCCH}$} & \multicolumn{2}{|c|}{ MS-NCCH } & \multicolumn{3}{|c|}{ MH-MBSP } \\
\hline & Result & Time & Avg & Time & Avg & Time & $\overline{\text { Gap }}$ \\
\hline $\operatorname{rn} 50 \mathrm{~d} 01-1$ & 39 & 0.00 & 41.0 & 0.06 & 41.0 & 0.07 & 0.00 \\
\hline $\operatorname{rn} 50 \mathrm{~d} 01-2$ & 35 & 0.00 & 40.0 & 0.01 & 40.0 & 0.14 & 0.00 \\
\hline rn50d01-3 & 36 & 0.00 & 43.0 & 0.06 & 43.0 & 0.07 & 0.00 \\
\hline rn50d03-1 & 18 & 0.00 & 33.0 & 0.08 & 33.0 & 0.09 & 0.00 \\
\hline rn50d03-2 & 32 & 0.00 & 33.0 & 0.08 & 33.0 & 0.09 & 0.00 \\
\hline rn50d03-3 & 31 & 0.00 & 32.9 & 0.03 & 32.9 & 0.04 & 0.10 \\
\hline rn50d05-1 & 27 & 0.00 & 30.0 & 0.08 & 30.0 & 0.09 & 0.00 \\
\hline rn50d05-2 & 27 & 0.00 & 28.0 & 0.05 & 28.0 & 0.06 & 0.00 \\
\hline rn50d05-3 & 29 & 0.00 & 30.0 & 0.08 & 30.0 & 0.09 & 0.00 \\
\hline rn50d07-1 & 23 & 0.00 & 26.0 & 0.07 & 26.0 & 0.08 & 0.00 \\
\hline $\operatorname{rn} 50 \mathrm{~d} 07-2$ & 24 & 0.00 & 27.0 & 0.08 & 27.0 & 0.09 & 0.00 \\
\hline $\operatorname{rn} 50 \mathrm{~d} 07-3$ & 11 & 0.00 & 24.9 & 0.02 & 24.9 & 0.05 & 0.10 \\
\hline rn100d01-1 & 60 & 0.00 & 75.0 & 0.33 & 75.0 & 0.40 & 0.00 \\
\hline rn100d01-2 & 52 & 0.00 & 72.0 & 0.16 & 72.0 & 0.42 & 0.00 \\
\hline rn100d01-3 & 54 & 0.00 & 78.0 & 0.32 & 78.0 & 0.37 & 0.00 \\
\hline rn100d03-1 & 40 & 0.00 & 54.0 & 0.29 & 54.0 & 0.35 & 0.00 \\
\hline rn100d03-2 & 46 & 0.00 & 56.0 & 0.19 & 56.0 & 0.21 & 0.00 \\
\hline rn100d03-3 & 40 & 0.00 & 50.0 & 0.08 & 50.0 & 0.52 & 0.00 \\
\hline rn100d05-1 & 38 & 0.00 & 43.9 & 0.04 & 44.0 & 0.09 & 0.00 \\
\hline rn100d05-2 & 21 & 0.00 & 41.0 & 0.14 & 41.0 & 1.16 & 0.00 \\
\hline rn100d05-3 & 13 & 0.00 & 42.0 & 0.07 & 42.0 & 1.73 & 0.00 \\
\hline rn100d07-1 & 31 & 0.00 & 38.4 & 0.28 & 39.0 & 1.77 & 0.00 \\
\hline rn100d07-2 & 36 & 0.00 & 39.1 & 0.22 & 39.1 & 0.34 & 0.90 \\
\hline rn100d07-3 & 26 & 0.00 & 36.3 & 0.14 & 36.6 & 2.06 & 0.40 \\
\hline rn150d01-1 & 71 & 0.00 & 96.0 & 0.87 & 96.0 & 1.18 & 0.00 \\
\hline rn150d01-2 & 91 & 0.00 & 103.0 & 0.80 & 103.0 & 0.83 & 0.00 \\
\hline rn150d01-3 & 93 & 0.00 & 103.0 & 0.85 & 103.0 & 0.87 & 0.00 \\
\hline rn150d03-1 & 58 & 0.00 & 71.0 & 0.24 & 71.0 & 0.30 & 0.00 \\
\hline rn150d03-2 & 54 & 0.00 & 67.0 & 0.45 & 67.0 & 1.26 & 0.00 \\
\hline rn150d03-3 & 41 & 0.00 & 67.8 & 0.31 & 68.0 & 2.64 & 0.00 \\
\hline rn150d05-1 & 40 & 0.00 & 55.5 & 0.26 & 56.0 & 5.77 & 0.00 \\
\hline rn150d05-2 & 46 & 0.00 & 55.7 & 0.11 & 55.9 & 1.88 & 0.10 \\
\hline rn150d05-3 & 30 & 0.00 & 52.2 & 0.26 & 53.0 & 5.64 & 0.00 \\
\hline rn150d07-1 & 25 & 0.00 & 45.1 & 0.28 & 45.3 & 20.61 & 0.70 \\
\hline rn150d07-2 & 25 & 0.00 & 46.8 & 0.17 & 47.0 & 8.68 & 0.00 \\
\hline rn150d07-3 & 31 & 0.00 & 44.8 & 0.20 & 44.9 & 8.22 & 1.10 \\
\hline rn200d01-1 & 79 & 0.00 & 119.0 & 0.56 & 119.0 & 0.79 & 0.00 \\
\hline rn200d01-2 & 121 & 0.00 & 131.0 & 1.88 & 131.0 & 1.92 & 0.00 \\
\hline rn200d01-3 & 102 & 0.00 & 121.0 & 0.76 & 121.0 & 0.83 & 0.00 \\
\hline rn200d03-1 & 59 & 0.00 & 74.3 & 0.26 & 74.9 & 3.46 & $*_{0.10}$ \\
\hline rn200d03-2 & 61 & 0.00 & 76.9 & 0.31 & 77.3 & 5.27 & 0.70 \\
\hline rn200d03-3 & 53 & 0.00 & 77.9 & 0.40 & 77.9 & 14.58 & $*_{0.10}$ \\
\hline rn200d05-1 & 45 & 0.00 & 59.4 & 0.25 & 59.4 & 26.76 & $* 0.60$ \\
\hline rn200d05-2 & 45 & 0.00 & 58.8 & 0.24 & 59.1 & 7.65 & $*_{-} 1.10$ \\
\hline rn200d05-3 & 46 & 0.00 & 61.7 & 0.29 & 61.7 & 22.00 & $*_{0} 0.30$ \\
\hline rn200d07-1 & 33 & 0.00 & 48.0 & 0.12 & 48.0 & 26.56 & $* 2.00$ \\
\hline rn200d07-2 & 28 & 0.00 & 48.9 & 0.15 & 48.9 & 22.31 & $*_{0.10}$ \\
\hline rn200d07-3 & 37 & 0.00 & 52.0 & 0.24 & 52.0 & 22.45 & $* 0.00$ \\
\hline
\end{tabular}


ran both heuristics in the same machine and executed each instance ten times with the same time limit as MH-MBSP (30 s).

Table 5 shows the results obtained for the set of instances DMERN. Columns 2 and 3 show the average GRASP solution and the gap between the best solution found by the exact method (ILP) and the average result found by the GRASP. Columns 4 and 6 have the same meaning as columns 2 and 3 but for the MH-MBSP matheuristic. Moreover, column 5 shows the average execution time for MH-MBSP, because the optimization phase could stop before the time limit. Those results in bold font are strictly better than the results from the other methods. An asterisk in the gap cells indicates that the exact method could not find an optimal solution in one hour, and the gap is calculated using the best solution found. The value "_" in the time cells indicates that the method reached the maximum assigned time of $30 \mathrm{~s}$.

Table 5 shows that the GRASP heuristic obtained the best average results for two instances, which are the smallest in the group and correspond to graphs with 144 vertices (danoint) and 184 vertices (bienst1). In these cases, the GRASP heuristic always found the optimal value, while the matheuristic MH-MBSP found the optimal value sometimes. For the 53 remaining instances, the MH-MBSP got better results than GRASP.

In general, as the instances' size increases, it is more difficult for GRASP to find good quality solutions in a limited time. It may happen because GRASP has a strictly quadratic and more intensive local search. Thus, for small instances, this more intensive search can find good values in a short time, but it would take much more time to achieve a better result for larger ones. In contrast, our local search explores a limited number of neighbors that is a fraction of the total number of vertices. Thus, we work with a local search with a linear behavior, which obtains good quality results for large instances without spending so much time.

We also analyzed the performance of the proposed methods on the Portfolio instances set, which is composed of instances with the number of vertices $|V|$ varying in the set $\{390,420,450,480,510\}$, and the threshold value $t$ used in the creation of the instances varying in the set $\{0.300,0.325,0.350,0.375,0.400\}$ (see Huffner et al. [14] for more details). For each combination of these values, there are ten different signed graphs, resulting in 250 instances.

Table 6 shows the results for the Portfolio instances. This table is presented in a format similar to that used by Figueiredo and Frota [8]. In this case, each line represents a group of 10 instances generated with the same threshold $t$. Columns 3-7 in this table have the same meaning as columns 2-6 in Table 5.

The matheuristics MH-MBSP always got a better result than the GRASP heuristic. Moreover, the value obtained by the matheuristic was very accurate as the gap column values are at a maximum distance of 1 from the optimal solution. In three groups of instances, the matheuristic MH-MBSP found all optimal values. For instances with 420 vertices and parameter $t=350$, it found a better solution than the one found by the exact algorithm in one hour of processing. Additionally, the average resolution time obtained by MH-MBSP was much less than the time limit of $30 \mathrm{~s}$.

In the last experiment, we consider the results obtained for the Random set of instances, which is composed by randomly generated instances classified into two groups. Group 1 is the set of random signed graphs without parallel edges $\left(E^{-} \cap E^{+}=\emptyset\right)$, with the number of vertices $|V|$ varying in the set $\{50,100,150,200\}$, density $d$ varying in the set $\{0.25,0.50,0.75\}$, and $\left|E^{-}\right| /\left|E^{+}\right|$varying in the set $\{0.5,1.0,2.0\}$. For each combination of these values, there are 3 different signed graphs, resulting in 108 instances. Group 2 is the set of random signed graphs with parallel edges $\left(E^{-} \cap E^{+} \neq \emptyset\right)$, with the number of vertices and the density varying as in Group 1 , and $\left|E^{-} \cap E^{+}\right| /|E|$ varying in the set $\{0.25,0.50,0.75\}$. For each combination of these values, there are 3 different signed graphs, resulting in other 108 instances.

Table 7 presents the results where each row represents a group of 27 graphs. Similarly to Table 6 , we give the average information per group of instances. For these instances, the GRASP heuristic obtained the best average results in all groups. However, the gaps were small for both heuristics, being at an average distance of only 1 from the best value obtained by the exact algorithm. We verified that the time used by the MS-NCCH heuristic is $0.6 \%$ of the time used by the exact phase of the MH-MBSP for these instances. We believe that if the local search of the MS-NCCH is more exhaustive for small instances like these, the elite set's quality may improve and accelerate the exact phase. 
TABle 5. Comparisons of heuristics methods on DMERN instances. For Tables 5-7, results in bold font indicate that are strictly better than the results from the other methods.

\begin{tabular}{|c|c|c|c|c|c|}
\hline \multirow[b]{2}{*}{ Instance } & \multicolumn{2}{|c|}{ GRASP } & \multicolumn{3}{|c|}{ MH-MBSP } \\
\hline & Avg & Gap & Avg & Time & Gap \\
\hline danoint & 97.0 & 0.00 & 96.4 & 0.45 & 0.60 \\
\hline bienst1 & 90.0 & 0.00 & 89.2 & 0.48 & 0.80 \\
\hline stein 45 & 30.0 & 0.00 & 30.0 & 0.62 & 0.00 \\
\hline disctom & 299.0 & 0.00 & 299.0 & 14.47 & 0.00 \\
\hline fc. 60.20 .1 & 366.2 & 4.80 & 371.0 & 0.35 & 0.00 \\
\hline air05 & 66.8 & 3.20 & 68.5 & 16.54 & $* 1.50$ \\
\hline neos 17 & 2.0 & 0.00 & 2.0 & 2.64 & 0.00 \\
\hline p100x588 & 624.2 & 8.80 & 633.0 & 2.21 & 0.00 \\
\hline air04 & 122.1 & 8.90 & 129.8 & - & $* 1.20$ \\
\hline r80x800 & 816.4 & 11.60 & 828.0 & 1.40 & 0.00 \\
\hline nug08 & 128.0 & 0.00 & 128.0 & 7.08 & 0.00 \\
\hline p $50 \times 864$ & 874.1 & 9.90 & 884.0 & 3.95 & 0.00 \\
\hline n5-3 & 686.2 & 225.80 & 912.0 & 6.14 & 0.00 \\
\hline neos 21 & 188.1 & 2.90 & 191.0 & 5.94 & 0.00 \\
\hline n4-3 & 804.6 & 257.40 & 1062.0 & 8.21 & 0.00 \\
\hline dano3mip & 629.3 & 140.70 & 649.0 & 17.73 & *121.00 \\
\hline $\mathrm{n} 8-3$ & 905.1 & 270.90 & 1176.0 & 9.31 & 0.00 \\
\hline neos 20 & 603.7 & 23.30 & 627.0 & 2.69 & 0.00 \\
\hline p200x1188 & 1250.3 & 22.70 & 1273.0 & 9.76 & 0.00 \\
\hline p200x1188c & 1249.8 & 26.20 & 1276.0 & 9.50 & 0.00 \\
\hline roll3000 & 794.0 & 30.00 & 824.0 & 13.71 & 0.00 \\
\hline janos-us-ca-D-D-M-N-C-A-N-N & 1188.8 & 332.20 & 1521.0 & 18.16 & 0.00 \\
\hline pioro40-D-B-M-N-C-A-N-N & 1306.5 & 253.50 & 1560.0 & 17.50 & 0.00 \\
\hline n13-3 & 1230.0 & 307.00 & 1537.0 & 25.46 & 0.00 \\
\hline $\mathrm{n} 2-3$ & 1359.3 & 296.70 & 1656.0 & 21.61 & 0.00 \\
\hline zib54-U-U-E-N-C-A-N-N & 1538.9 & 189.10 & 1728.0 & 23.95 & 0.00 \\
\hline qap10 & 200.0 & 0.00 & 200.0 & - & 0.00 \\
\hline ns1688347 & 374.0 & 74.00 & 444.7 & - & *3.30 \\
\hline germany50-U-U-M-N-C-A-N-N & 1660.5 & 339.50 & 2000.0 & - & 0.00 \\
\hline protfold & 403.2 & 169.80 & 546.0 & 12.19 & $* 27.00$ \\
\hline cap6000 & 2074.0 & 0.00 & 2074.0 & - & 0.00 \\
\hline $\mathrm{n} 7-3$ & 1750.3 & 411.70 & 2162.0 & - & 0.00 \\
\hline n9-3 & 1433.5 & 678.50 & 2112.0 & - & 0.00 \\
\hline acc-1 & 380.5 & 110.50 & 456.0 & - & 35.00 \\
\hline n3-3 & 1382.4 & 676.60 & 2059.0 & - & 0.00 \\
\hline zib54-D-B-E-N-C-A-N-N & 1985.5 & 282.50 & 2268.0 & - & 0.00 \\
\hline n12-3 & 1715.5 & 498.50 & 2214.0 & - & 0.00 \\
\hline neos 818918 & 1753.4 & 264.60 & 2018.0 & 2.34 & 0.00 \\
\hline germany50-D-B-M-N-C-A-N-N & 1857.0 & 493.00 & 2350.0 & - & 0.00 \\
\hline acc-2 & 375.5 & 115.50 & 450.9 & - & 40.10 \\
\hline ta2-U-U-M-N-C-A-N-N & 2036.0 & 435.00 & 2471.0 & - & 0.00 \\
\hline n6-3 & 2099.9 & 438.10 & 2538.0 & - & 0.00 \\
\hline berlin & 1670.5 & 982.50 & 2653.0 & - & 0.00 \\
\hline neos11 & 637.6 & 104.40 & 741.7 & 9.50 & 0.30 \\
\hline ta2-D-B-M-N-C-A-N-N & 2097.2 & 633.80 & 2731.0 & - & 0.00 \\
\hline acc-6 & 776.4 & 200.60 & 952.1 & - & 24.90 \\
\hline acc-5 & 787.6 & 190.40 & 952.9 & - & 25.10 \\
\hline acc-3 & 852.3 & 272.70 & 1125.0 & 21.41 & 0.00 \\
\hline
\end{tabular}


TABLE 5. continued.

\begin{tabular}{lrrrrrr}
\hline & \multicolumn{3}{c}{ GRASP } & & \multicolumn{3}{c}{ MH-MBSP } \\
\cline { 2 - 3 } \cline { 6 - 7 } Instance & Avg & Gap & & Avg & Time & Gap \\
\hline acc-4 & 852.4 & 272.60 & $\mathbf{1 1 2 5 . 0}$ & 20.74 & 0.00 \\
brasil & 1044.2 & 2262.80 & $\mathbf{3 3 0 7 . 0}$ & - & 0.00 \\
mkc & 1575.5 & 1647.50 & $\mathbf{3 2 2 3 . 0}$ & 23.37 & 0.00 \\
p500x2988 & 1081.9 & 2124.10 & $\mathbf{3 2 0 6 . 0}$ & - & 0.00 \\
p500x2988c & 1077.3 & 2116.70 & $\mathbf{3 1 9 4 . 0}$ & 11.86 & 0.00 \\
mod011 & 1822.4 & 2031.60 & $\mathbf{3 8 5 4 . 0}$ & - & 0.00 \\
neos1 & 936.0 & 250.00 & $\mathbf{1 1 2 7 . 6}$ & - & 58.40 \\
seymour & 541.0 & 69.00 & $\mathbf{5 8 2 . 0}$ & - & $* 28.00$ \\
seymour1 & 543.1 & 66.90 & $\mathbf{5 8 1 . 0}$ & - & $* 29.00$ \\
n370a & 457.7 & 4642.30 & $\mathbf{5 1 0 0 . 0}$ & - & 0.00 \\
rentacar & 2109.1 & 2583.90 & $\mathbf{4 6 8 4 . 8}$ & 20.89 & 8.20 \\
manna81 & 336.8 & 1985.20 & $\mathbf{2 3 2 2 . 0}$ & - & 0.00 \\
neos12 & 219.9 & 1326.10 & $\mathbf{1 5 0 5 . 4}$ & - & 40.60 \\
\hline
\end{tabular}

TABle 6. Comparisons of heuristics methods on Portfolio instances.

\begin{tabular}{lrrrrrr}
\hline \hline & & \multicolumn{2}{c}{ GRASP } & \multicolumn{3}{c}{ MH-MBSP } \\
\cline { 3 - 6 }$V$ & $t$ & Avg & Gap & Avg & Time & Gap \\
\hline 390 & 0.300 & 255.7 & 29.53 & $\mathbf{2 8 4 . 3}$ & 1.8 & ${ }^{*} 0.93$ \\
& 0.325 & 282.3 & 33.45 & $\mathbf{3 1 5 . 2}$ & 1.3 & ${ }^{*} 0.55$ \\
& 0.350 & 309.2 & 34.12 & $\mathbf{3 4 3 . 1}$ & 1.5 & 0.20 \\
& 0.375 & 344.7 & 22.42 & $\mathbf{3 6 6 . 3}$ & 1.3 & 0.78 \\
& 0.400 & 373.5 & 7.76 & $\mathbf{3 8 1 . 2}$ & 1.5 & 0.12 \\
420 & 0.300 & 276.1 & 23.41 & $\mathbf{2 9 8 . 9}$ & 2.5 & ${ }^{*} 0.63$ \\
& 0.325 & 299.9 & 32.57 & $\mathbf{3 3 2 . 3}$ & 2.0 & ${ }^{*} 0.23$ \\
& 0.350 & 327.3 & 37.70 & $\mathbf{3 6 5 . 0}$ & 1.6 & ${ }^{*}-0.01$ \\
& 0.375 & 356.4 & 34.78 & $\mathbf{3 9 1 . 1}$ & 0.7 & 0.10 \\
450 & 0.400 & 400.4 & 6.77 & $\mathbf{4 0 7 . 2}$ & 1.1 & 0.00 \\
& 0.300 & 285.2 & 32.69 & $\mathbf{3 1 7 . 5}$ & 2.4 & ${ }^{*} 0.37$ \\
& 0.325 & 314.0 & 40.02 & $\mathbf{3 5 3 . 7}$ & 2.1 & ${ }^{*} 0.28$ \\
& 0.350 & 356.7 & 33.20 & $\mathbf{3 8 9 . 8}$ & 1.5 & ${ }^{*} 0.08$ \\
& 0.375 & 383.4 & 35.02 & $\mathbf{4 1 8 . 4}$ & 0.8 & 0.00 \\
480 & 0.400 & 415.9 & 20.29 & $\mathbf{4 3 6 . 2}$ & 1.0 & 0.00 \\
& 0.300 & 300.7 & 33.24 & $\mathbf{3 3 3 . 4}$ & 4.3 & ${ }^{*} 0.52$ \\
& 0.325 & 325.9 & 46.67 & $\mathbf{3 7 1 . 4}$ & 3.8 & ${ }^{*} 1.18$ \\
& 0.350 & 357.3 & 52.17 & $\mathbf{4 0 9 . 2}$ & 2.7 & ${ }^{*} 0.33$ \\
& 0.375 & 393.4 & 48.29 & $\mathbf{4 4 1 . 7}$ & 1.9 & 0.03 \\
510 & 0.400 & 428.9 & 34.82 & $\mathbf{4 6 3 . 6}$ & 2.2 & 0.08 \\
& 0.300 & 316.4 & 32.71 & $\mathbf{3 4 9 . 1}$ & 7.0 & ${ }^{*} 0.01$ \\
& 0.325 & 344.6 & 45.24 & $\mathbf{3 8 9 . 3}$ & 5.8 & ${ }^{*} 0.54$ \\
& 0.350 & 374.2 & 55.02 & $\mathbf{4 2 8 . 1}$ & 3.9 & ${ }^{*} 1.13$ \\
& 0.375 & 423.0 & 41.41 & $\mathbf{4 6 4 . 3}$ & 2.4 & ${ }^{*} 0.07$ \\
& 0.400 & 449.1 & 42.29 & $\mathbf{4 9 1 . 4}$ & 3.4 & 0.00 \\
\hline
\end{tabular}


TABLE 7. Comparisons of heuristics methods on Random instances.

\begin{tabular}{|c|c|c|c|c|c|c|}
\hline & \multirow[b]{2}{*}{$V$} & \multicolumn{2}{|c|}{ GRASP } & \multicolumn{3}{|c|}{ MH-MBSP } \\
\hline & & Avg & Gap & Avg & Time & Gap \\
\hline \multirow[t]{4}{*}{ Group 1} & 50 & 17.89 & 0.000 & 17.74 & 0.60 & 0.153 \\
\hline & 100 & 23.51 & 0.005 & 23.24 & 18.87 & ${ }^{*} 0.389$ \\
\hline & 150 & 26.43 & 0.010 & 25.88 & 25.78 & $*^{*} 0.819$ \\
\hline & 200 & 28.14 & 0.013 & 27.80 & 26.81 & ${ }^{*} 0.716$ \\
\hline \multirow[t]{4}{*}{ Group 2} & 50 & 12.44 & 0.000 & 12.18 & 0.21 & 0.267 \\
\hline & 100 & 16.14 & 0.000 & 15.94 & 9.31 & $*^{*} 0.209$ \\
\hline & 150 & 18.17 & 0.003 & 17.79 & 17.84 & $* 0.435$ \\
\hline & 200 & 19.02 & 0.001 & 18.71 & 22.66 & $* 0.329$ \\
\hline
\end{tabular}

TABLE 8. Instances and targets for convergence analysis.

\begin{tabular}{lrr}
\hline \hline Instance & $|V|$ & Target \\
\hline air05 & 426 & 66 \\
air04 & 823 & 120 \\
ns1688347 & 1866 & 370 \\
\hline
\end{tabular}

We can conclude from Tables 5-7 that, up to 200 vertices, the GRASP and MH-MBSP heuristics are quite similar in terms of the results' quality. But for instances with more than 200 vertices, the heuristic MH-MBSP always achieved better results, both in terms of solution quality and CPU time.

For the last experiment, we evaluated and compared the run-time distributions (also know as time-to-target plots) of the GRASP heuristic and the MH-MBSP matheuristic for some instances. Time-to-target plots display on the ordinate axis the probability that an algorithm will find a solution at least as good as a given target value in a given running time, shown on the abscissa axis.

The heuristics were performed 200 times in each instance, with different seeds for the pseudo-random number generator. Then, the empirical probability distributions of each heuristic time spent to find a target solution value are plotted. The methodology described in [1] was adopted to trace each heuristic's empirical distribution. The run times $\left(t_{i}\right)$ are sorted in ascending order, and a probability $p_{i}=\left(i-\frac{1}{2}\right) / 200$ is associated with each $t_{i}$. Then, the $z_{i}=\left(t_{i}, p_{i}\right)$ points for $i=1, \ldots, 200$ are plotted. The best algorithm is the one that presents the corresponding leftmost plots.

We chose two instances from different sizes to analyze the performance of the methods (air05 and ns1688347). Table 8 shows the instance's name, size, and target value. Both heuristics reached all chosen target values in the previous experiments since our interest was the convergence analysis.

Figure 1 shows the cumulative probability for the MH-MBSP for the instance air05, while Figure 2 shows the cumulative probability for both heuristics. In Figure 1, we can see how MH-MBSP reaches the target value very fast, with a high probability of finding the target in a time close to $0.16 \mathrm{~s}$. Also, compared with the GRASP heuristic, the figure shows that GRASP needs much more time to reach the target value. In addition, for a probability of finding the target value greater than 50\%, the GRASP heuristic needs at least $50 \mathrm{~s}$.

Figures 3 and 4 show the cumulative probability for the $n s 1688347$ instance. Again, the MH-MBSP quickly reaches (less than one second) the target value for all iterations. While Figure 4 shows that GRASP presents a probability of $70 \%$ of reaching the target value. According to the obtained results, the minimum time to find the target solution for this instance by the GRASP heuristic was $3.3 \mathrm{~s}$. Moreover, it was necessary to use a time close to $8.8 \mathrm{~s}$ to reach the target value with a probability greater than $50 \%$. 


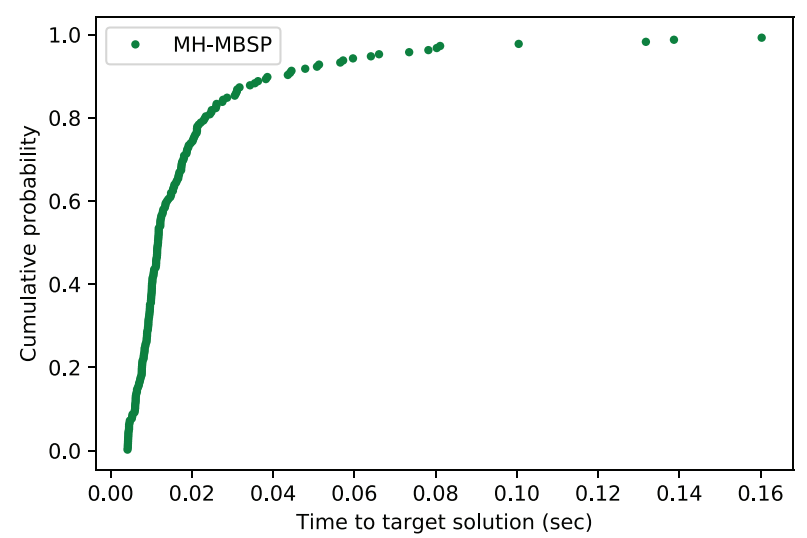

FiguRE 1. Cumulative probability of MH-MBSP for air05 instance.

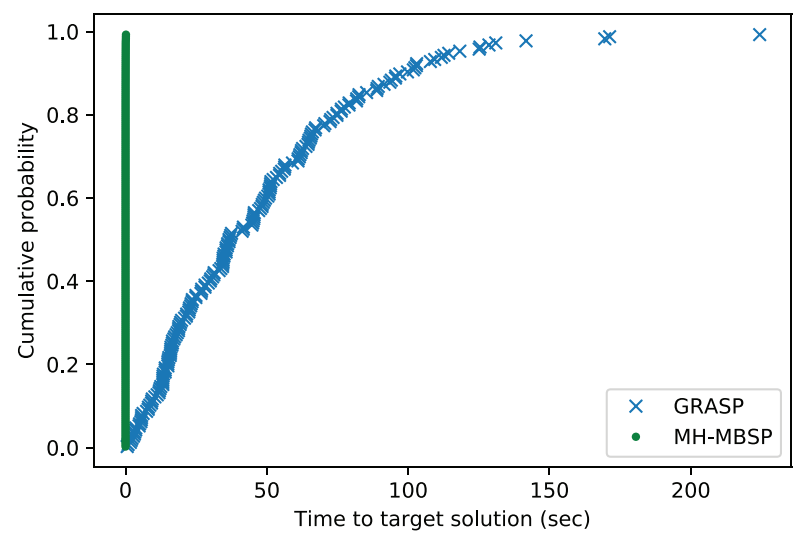

Figure 2. Cumulative probability of MH-MBSP and GRASP for air05 instance.

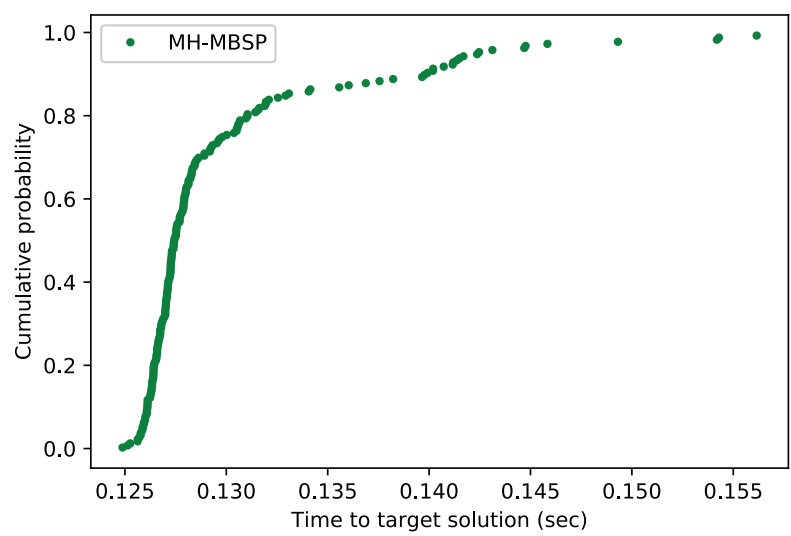

FiguRE 3. Cumulative probability of MH-MBSP for $n s 1688347$ instance. 


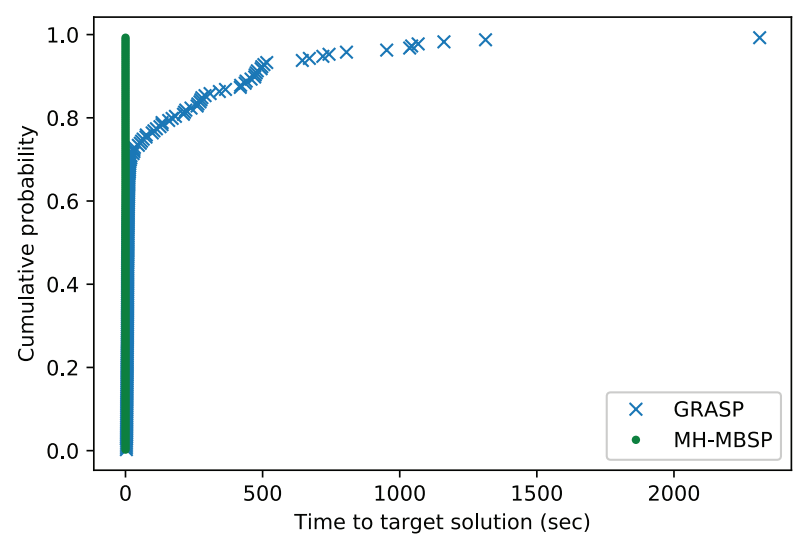

Figure 4. Cumulative probability of MH-MBSP and GRASP for $n s 1688347$ instance.

\section{Conclusions}

In this work, we developed methods to obtain heuristic solutions for the MBSP problem. We also provided an alternative formulation for the problem based on the basic definition of a balanced signed graph, whose size grows polynomially as a function of the input size.

Our main contribution in this work is the development of a matheuristic for the MBSP problem. Using different strategies as a multi-start scheme and a fast local search combined with an exact optimization phase allowed our method to obtain quickly good quality solutions for the tested sets of instances.

The results show that the proposed matheuristic is an appropriate choice to find good quality solutions for the MBSP problem. Furthermore, for large instances (with more than 200 vertices), the matheuristic MH_MBSP achieved in $30 \mathrm{~s}$ similar or better results than those obtained by solving exactly the problem using the proposed formulation in one hour of processing.

Our current research focus on the investigation of the structure of the polynomial size formulation in order to develop robust and efficient exact methods for the MBSP. Another direction for future research involves extending the solution method to similar balanced subgraph problems, such as KMBSP, where the goal is to find $k$-balanced components in the graph.

Acknowledgements. This study was financed in part by Fundação Carlos Chagas Filho de Amparo à Pesquisa do Estado de Rio de Janeiro and Conselho Nacional de Desenvolvimento Científico e Tecnológico. The authors would also like to thank the anonymous reviewer, who helped improve the manuscript with constructive comments.

\section{REFERENCES}

[1] R.M. Aiex, M.G.C. Resende and C.C. Ribeiro, Ttt plots: a perl program to create time-to-target plots. Optim. Lett. 1 (2007) $355-366$.

[2] F. Barahona and A.R. Mahjoub, Facets of the balanced (acyclic) induced subgraph polytope. Math. Program. 45 (1989) 21-33.

[3] M. Birattari, Z. Yuan, P. Balaprakash and T. Stützle, F-race and iterated f-race: An overview. In: Experimental methods for the analysis of optimization algorithms. Springer (2010) 311-336.

[4] D. Cartwright and F. Harary, Structural balance: a generalization of heider's theory. Psychol. Rev. 63 (1956) 277.

[5] B. DasGupta, G. Enciso, E. Sontag and Y. Zhang, Algorithmic and complexity results for decompositions of biological networks into monotone subsystems. Biosystems 90 (2006) 161-178.

[6] M.R. de Holanda Maia, A. Plastino and P.H.V. Penna, Minereduce: an approach based on data mining for problem size reduction. Comput. Oper. Res. 122 (2020) 104995.

[7] N. Dupin and E.-G. Talbi, Parallel matheuristics for the discrete unit commitment problem with minstop ramping constraints. Int. Trans. Oper. Res. 27 (2020) 219-244. 
[8] R. Figueiredo and Y. Frota, The maximum balanced subgraph of a signed graph: Applications and solution approaches. Eur. J. Oper. Res. 236 (2014) 473-487.

[9] R.M.V. Figueiredo, M. Labbé and C.C. De Souza, An exact approach to the problem of extracting an embedded network matrix. Comput. Oper. Res. 38 (2011) 1483-1492.

[10] M. Fischetti and M. Fischetti, Matheuristics. In: Handbook of Heuristics. Springer (2018) 121-153.

[11] N. Gülpinar, G. Gutin, G. Mitra and A. Zverovitch, Extracting pure network submatrices in linear programs using signed graphs. Discrete Appl. Math. 137 (2004) 359-372.

[12] F. Harary, M.-H. Lim and D.C. Wunsch, Signed graphs for portfolio analysis in risk management. IMA J. Manage. Math. 13 (2002) 201-210.

[13] F. Heider, Attitudes and cognitive organization. J. Psychology 21 (1946) 107-112.

[14] F. Hüffner, N. Betzler and R. Niedermeier, Separator-based data reduction for signed graph balancing. J. Comb. Optim. 20 (2010) 335-360.

[15] M. López-Ibáñez, J. Dubois-Lacoste, L.P. Cáceres, M. Birattari and T. Stützle, The irace package: Iterated racing for automatic algorithm configuration. Oper. Res. Perspect. 3 (2016) 43-58.

[16] F. Marinelli and A. Parente, A heuristic based on negative chordless cycles for the maximum balanced induced subgraph problem. Comput. Oper. Res. 69 (2016) 68-78.

[17] B. Martın, Á. Sánchez, C. Beltran-Royo and A. Duarte, A matheuristic approach for solving the edge-disjoint paths problem. Matheuristics 2016 (2016) 25.

[18] D. Martins, G.M. Vianna, I. Rosseti, S.L. Martins and A. Plastino, Making a state-of-the-art heuristic faster with data mining. Ann. Oper. Res. 263 (2018) 141-162.

[19] M.G.C. Resende and C.C. Ribeiro, Greedy randomized adaptive search procedures: advances and extensions. In: Handbook of metaheuristics. Springer (2019) 169-220.

[20] S. Wolfram, Wolfram Research. Inc., Mathematica, Version 8 (2013) 23.

[21] T. Zaslavsky, A mathematical bibliography of signed and gain graphs and allied areas. Electron. J. Comb. 1000 (2012) DS8.

\section{Subscribe to Open (S2O) A fair and sustainable open access model}

This journal is currently published in open access under a Subscribe-to-Open model (S2O). S2O is a transformative model that aims to move subscription journals to open access. Open access is the free, immediate, online availability of research articles combined with the rights to use these articles fully in the digital environment. We are thankful to our subscribers and sponsors for making it possible to publish this journal in open access, free of charge for authors.

\section{Please help to maintain this journal in open access!}

Check that your library subscribes to the journal, or make a personal donation to the S2O programme, by contacting subscribers@edpsciences.org

More information, including a list of sponsors and a financial transparency report, available at: https://www. edpsciences.org/en/maths-s2o-programme 